\title{
ON QUADRATURE FORMULAS FOR SINGULAR INTEGRAL EQUATIONS OF THE FIRST AND THE SECOND KIND*
}

\author{
$\mathrm{BY}$ \\ STEEN KRENK \\ Technical University of Denmark
}

\begin{abstract}
In this paper it is shown that by proper choice of the collocation points singular integral equations of the first and the second kind can be integrated by use of the usual Gauss-Jacobi quadrature formula. Detailed formulas are given for various values of the index.
\end{abstract}

1. Introduction. A large number of mixed boundary-value problems can be reduced to the problem of solving a system of singular integral equations of the form

$$
\begin{aligned}
a_{i} \phi_{i}(x)+\frac{b_{i}}{\pi} \int_{-1}^{1} \phi_{i}(t) \frac{d t}{t-x}+\sum_{i=1}^{R} \int_{-1}^{1} k_{i i}(x, t) \phi_{i}(t) d t & =g_{i}(x), \\
-1<x & <1, \quad i=1, \cdots, R .
\end{aligned}
$$

In (1.1) the $a_{i} \mathrm{~s}$ and $b_{i} \mathrm{~s}$ are real constants, and the kernels $k_{i j}(x, t)$ are bounded in the closed domain $-1 \leq(x, t) \leq 1$. The $g_{i}$ are known functions and the functions $\phi_{i}$ are the unknowns of the problem. At the endpoints $x= \pm 1$ the $\phi_{i}$ S or their first derivatives have integrable singularities.

A general closed-form solution to (1.1) is not known, but in [1] and [2] numerical methods are outlined. By applying the method given in [2] and [3] a group of fundamental functions which characterize the singular behavior of $\phi_{i}$ is found to be

$$
\begin{aligned}
& w_{i}(x)=(1-x)^{\alpha i}(1+x)^{\beta i}, \\
& \alpha_{i}=\frac{1}{2 \pi i} \log \left(\frac{a_{j}-i b_{i}}{a_{i}+i b_{j}}\right)+N_{i}, \\
& \beta_{i}=-\frac{1}{2 \pi i} \log \left(\frac{a_{i}-i b_{i}}{a_{i}+i b_{i}}\right)+M_{i} .
\end{aligned}
$$

$M_{i}$ and $N_{i}, j=1,2, \cdots, R$ are integers and for each of the $R$ equations in (1.1) the index is defined as

$$
\kappa_{i}=-\left(\alpha_{i}+\beta_{i}\right)=-\left(N_{i}+M_{i}\right) \quad j=1, \cdots, R .
$$

In order to get integrable singularities $\kappa$ must be restricted to $-1,0,1$.

* Received October 19, 1973, revised version received March 25, 1974. The work presented in this paper was carried out during a visit to Lehigh University and was supported in part by the National Science Foundation under the Grant GK-11977. 
To obtain a solution by numerical means the following expansions are used:

$$
\begin{aligned}
\phi_{i}(x) & =f_{i}(x) w_{i}(x), \\
f_{i}(x) & =\sum_{i=0}^{\infty} B_{i i} P_{i}^{(\alpha, \beta)}(x) .
\end{aligned}
$$

In (1.6) $P_{i}{ }^{(\alpha, \beta)}(x)$ is the Jacobi polynomial of degree $j$ with indices $\alpha$ and $\beta$. In [2] the expansion (1.6) is used in connection with the orthogonality properties of the Jacobi polynomials. An infinite system of equations results, and by truncation an approximate solution is obtained. For $a_{i}=0, i=1, \cdots, R$, an alternative method is available. In [1] and [2] it is proved that for this special case quadrature formulas of Gauss-Chebyshev type exist for the singular integral. This means that for proper selection of $x$ all integrals can be evaluated by the usual quadrature formulas for continuous bounded functions.

In this paper the latter method is extended to the general case, and it is demonstrated how one obtains the formulas from [1] and [2] as special cases. Only a single equation is considered, as the extension to a system of equations of the form (1.1) is trivial.

2. A quadrature formula. As a basis for the calculations the following result from [2] and [4] is used:

$$
\begin{gathered}
a w(x) P_{i}{ }^{(\alpha, \beta)}(x)+\frac{b}{\pi} \int_{-1}^{1} w(t) P_{i}{ }^{(\alpha, \beta)}(t) \frac{d t}{t-x}=-2^{-\kappa} \frac{\Gamma(\alpha) \Gamma(1-\alpha)}{\pi} b P_{i-\kappa}{ }^{(-\alpha,-\beta)}(x), \\
-1<x<1 .
\end{gathered}
$$

It is noted that, while the two terms on the left-hand side of (2.1) are both singular, their sum is regular. From the Jacobi polynomials another type of polynomials is formed in the following way:

$$
Q_{n, j}{ }^{(\alpha, \beta)}(x)=P_{n-\kappa}{ }^{(-\alpha,-\beta)}(x) P_{i}^{(\alpha, \beta)}(x)-P_{n}^{(\alpha, \beta)}(x) P_{i-\kappa}{ }^{(-\alpha,-\beta)}(x) .
$$

Provided $Q_{n, j}{ }^{(\alpha, \beta)}(x)$ is of degree equal to or less than $n$, the following expansion is possible:

$$
\frac{P_{n-\kappa}{ }^{(-\alpha,-\beta)}(x) P_{j}^{(\alpha, \beta)}(x)-P_{n}^{(\alpha, \beta)}(x) P_{j-\kappa}{ }^{(-\alpha,-\beta)}(x)}{P_{n}^{(\alpha, \beta)}(x)}=-\sum_{i=1}^{n} \frac{c_{i}}{t_{i}-x}
$$

where $t_{2}$ is determined by

$$
P_{n}^{(\alpha, \beta)}\left(t_{i}\right)=0, \quad i=1,2, \cdots, n .
$$

In the next section it is proved that $Q_{n, j}{ }^{(\alpha, \beta)}(x)$ is of degree $n-j-1$ for $\kappa=-10,1$ and $n \geq j$. The expansion coefficients $c_{i}$ are then determined by

$$
c_{i}=\frac{P_{n-\kappa}{ }^{(-\alpha,-\beta)}\left(t_{i}\right) P_{i}^{(\alpha, \beta)}\left(t_{i}\right)}{P_{n}^{(\alpha, \beta)}\left(t_{i}\right)} .
$$

By choosing $x_{k}$ as

$$
P_{n-\kappa}^{(-\alpha,-\beta)}\left(x_{k}\right)=0, \quad k=1,2, \cdots, n-\kappa,
$$

(2.3) and (2.5) yield

$$
P_{i-k}{ }^{(-\alpha,-\beta)}\left(x_{k}\right)=\sum_{i=1}^{n} \frac{P_{n-k}{ }^{(-\alpha,-\beta)}\left(t_{i}\right)}{P_{n}^{(\alpha, \beta)}\left(t_{i}\right)} \frac{P_{i}^{(\alpha, \beta)}\left(t_{i}\right)}{t_{i}-x_{k}} .
$$


Now (2.7) is used to evaluate the expression

$$
S(x)=\frac{a}{b} \phi(x)+\frac{1}{\pi} \int_{-1}^{1} \phi(t) \frac{d t}{t-x}
$$

where

$$
\phi(x)=f(x) w(x) .
$$

and $f(x)$ is approximated by a truncated series of the form (1.6)

$$
\tilde{f}(x)=\sum_{i=0}^{p} B_{i} P_{i}^{(\alpha, \beta)}(x) .
$$

Using (2.9), (2.10), (2.1) and (2.7), we obtain

$$
\begin{aligned}
\tilde{S}\left(x_{k}\right) & =-2^{-\kappa} \frac{\Gamma(\alpha) \Gamma(1-\alpha)}{\pi} \sum_{i=0}^{p} B_{i} P_{i-\kappa}{ }^{(-\alpha,-\beta)}\left(x_{k}\right) \\
& =-2^{-\kappa} \frac{\Gamma(\alpha) \Gamma(1-\alpha)}{\pi} \sum_{i=0}^{p} \sum_{i=1}^{n} \frac{P_{n-\kappa}{ }^{(-\alpha,-\beta)}\left(t_{i}\right)}{P_{n}^{(\alpha, \beta)^{\prime}}\left(t_{i}\right)} \frac{B_{i} P_{i}{ }^{(\alpha, \beta)}\left(t_{i}\right)}{t_{i}-x_{k}} \\
& =-2^{-\kappa} \frac{\Gamma(\alpha) \Gamma(1-\alpha)}{\pi} \sum_{i=1}^{n} \frac{P_{n-\kappa}{ }^{(-\alpha,-\beta)}\left(t_{i}\right)}{P_{n}^{(\alpha, \beta)^{\prime}}\left(t_{i}\right)} \frac{\tilde{f}\left(t_{i}\right)}{t_{i}-x_{k}} .
\end{aligned}
$$

Two points require notice in (2.11). First: the only approximation involved is the truncation of the series (2.10). Second: it is necessary to use $p \leq n$, which is equivalent to using $j \leq n$ for all $j$.

In (2.11) $\widetilde{S}\left(x_{k}\right)$ is calculated by a procedure analogous to mechanical quadrature of an integral, but it must be kept in mind that $S\left(x_{k}\right)$ consists of an integral plus a nonintegral term.

(2.11) is of the form

$$
S\left(x_{k}\right) \simeq \sum_{i=1}^{n} W_{i}^{n} \frac{f\left(t_{i}\right)}{t_{i}-x_{k}}
$$

with the weights given by

$$
W_{i}^{n}=-2^{-\kappa} \frac{\Gamma(\alpha) \Gamma(1-\alpha)}{\pi} \frac{P_{n-\kappa}{ }^{(-\alpha,-\beta)}\left(t_{i}\right)}{P_{n}^{(\alpha, \beta)^{\prime}}\left(t_{i}\right)} .
$$

$W_{i}^{n}$ will be put in standard form in Sec. 4 .

3. The polynomials $Q_{n, i}{ }^{(\alpha, \beta)}(x)$. The polynomials $Q_{n, i}{ }^{(\alpha, \beta)}(x)$ are defined by $(2.2)$

$$
Q_{n, j}{ }^{(\alpha, \beta)}(x)=P_{n-\kappa}{ }^{(-\alpha,-\beta)}(x) P_{i}^{(\alpha, \beta)}(x)-P_{n}^{(\alpha, \beta)}(x) P_{i-\kappa}^{(-\alpha,-\beta)}(x) .
$$

For $n=j(3.1)$ yields

$$
Q_{i, i}^{(\alpha, \beta)}(x) \equiv 0 .
$$

The properties of $Q_{n, i}{ }^{(\alpha, \beta)}(x)$ are established by use of the recurrence relation for the Jacobi polynomials [5].

$$
\begin{aligned}
a_{1, n}{ }^{(\alpha, \beta)} P_{n+1}{ }^{(\alpha, \beta)}(x) & =\left[a_{2, n}{ }^{(\alpha, \beta)}+x a_{3, n}{ }^{(\alpha, \beta)}\right] P_{n}^{(\alpha, \beta)}(x) \\
& -a_{4, n}{ }^{(\alpha, \beta)} P_{n-1}{ }^{(\alpha, \beta)}(x), \quad n \geq 0, \\
a_{1, n}{ }^{(\alpha, \beta)} & =2(n+1)(n+\alpha+\beta+1)(2 n+\alpha+\beta),
\end{aligned}
$$




$$
\begin{aligned}
& a_{2, n}{ }^{(\alpha, \beta)}=(2 n+\alpha+\beta+1)\left(\alpha^{2}-\beta^{2}\right), \\
& a_{3, n}{ }^{(\alpha, \beta)}=(2 n+\alpha+\beta)(2 n+\alpha+\beta+1)(2 n+\alpha+\beta+2), \\
& a_{4, n}{ }^{(\alpha, \beta)}=2(n+\alpha)(n+\beta)(2 n+\alpha+\beta+2) .
\end{aligned}
$$

From (3.3) the following relations are obtained:

$$
a_{i, n-\kappa}{ }^{(-\alpha,-\beta)}=a_{i, n}{ }^{(\alpha, \beta)}, \quad i=1,2,3,4 .
$$

By using (3.4) in (3.1) a recurrence relation for $Q_{n, j}{ }^{(\alpha, \beta)}(x)$ is established:

$a_{1, n}{ }^{(\alpha, \beta)} Q_{n+1, i}{ }^{(\alpha, \beta)}(x)=\left[a_{2, n}{ }^{(\alpha, \beta)}+x a_{3, n}{ }^{(\alpha, \beta)}\right] Q_{n, i}{ }^{(\alpha, \beta)}(x)-a_{4, n}{ }^{(\alpha, \beta)} Q_{n-1, i}{ }^{(\alpha, \beta)}(x)$.

For $n=j$ (3.5) yields

$a_{1, j}{ }^{(\alpha, \beta)} Q_{i+1, i}{ }^{(\alpha, \beta)}(x)=-a_{4, n}{ }^{(\alpha, \beta)} Q_{i-1, i}{ }^{(\alpha, \beta)}(x)=a_{4, n}{ }^{(\alpha, \beta)} Q_{j, j-1}{ }^{(\alpha, \beta)}(x)$.

By continued use of (3.6) formulas for $Q_{i+1, j}{ }^{(\alpha, \beta)}(x)$ are obtained. For $\kappa=-1,0,1$ these have the common form

$$
Q_{i+1, j}{ }^{(\alpha, \beta)}(x)=-\frac{\Gamma(j+1+\alpha) \Gamma(j+1+\beta)}{\Gamma(j+\alpha+\beta+2)(j+1) !} \frac{2 j+\alpha+\beta+2}{\Gamma(\alpha) \Gamma(1-\alpha)} .
$$

From (3.2), (3.7) and the recurrence relation (3.5) it follows by induction that $Q_{n, j}{ }^{(\alpha, \beta)}(x)$ is a polynomial of degree $n-j-1$ for $\kappa=-1,0,1$ and $n \geq j$.

4. The weights $W_{i}{ }^{n}$. The weights $W_{i}{ }^{n}$ are given by (2.13). By using (3.1) $P_{n-\kappa}^{(-\alpha,-\beta)}\left(t_{i}\right)$ is eliminated:

$$
P_{n-\kappa}{ }^{(-\alpha,-\beta)}\left(t_{i}\right)=\frac{Q_{n, i}{ }^{(\alpha, \beta)}\left(t_{i}\right)}{P_{i}^{(\alpha, \beta)}\left(t_{i}\right)} .
$$

Two values of $j$ are of practical interest. For $j=n-1$ (4.1) and (2.13) yield

$$
W_{i}^{n}=\frac{1}{\pi} 2^{(\alpha+\beta)} \frac{\Gamma(n+\alpha) \Gamma(n+\beta)}{n ! \Gamma(n+\alpha+\beta+1)} \frac{2 n+\alpha+\beta}{P_{n}^{(\alpha, \beta)}\left(t_{i}\right) P_{n-1}{ }^{(\alpha, \beta)}\left(t_{i}\right)},
$$

while $j=n+1$ leads to the laternative formula

$$
W_{i}^{n}=-\frac{1}{\pi} 2^{(\alpha+\beta)} \frac{\Gamma(n+1+\alpha) \Gamma(n+1+\beta)}{(n+1) ! \Gamma(n+\alpha+\beta+2)} \frac{2 n+\alpha+\beta+2}{P_{n}^{(\alpha, \beta)}\left(t_{i}\right) P_{n+1}{ }^{(\alpha, \beta)}\left(t_{i}\right)} .
$$

It is noted that (4.2) and (4.3), with the exception of the factor $1 / \pi$, are the GaussJacobi weights for integration of continuous bounded functions $[2,6]$.

For $a=0$ (1.3) yields $\alpha= \pm \frac{1}{2}$ and $\beta= \pm \frac{1}{2}$. When this is the case the quadrature formulas may be obtained solely by means of trigonometric formulas and explicit expressions for $t_{i}$ and $x_{k}$ are obtained. These special formulas are given in [1] and [2].

5. Solution of a singular integral equation. The following singular integral equation is considered:

$$
\begin{gathered}
a \phi(x)+\frac{b}{\pi} \int_{-1}^{1} \phi(t) \frac{d t}{t-x}+\int_{-1}^{1} k(x, t) \phi(t) d t=g(x), \quad-1<x<1, \\
\phi(x)=f(x)(1-x)^{\alpha}(1+x)^{\beta} .
\end{gathered}
$$


5.1 The case of $\kappa=1$. For this case $\alpha<0$ and $\beta<0$. The problem consists of (5.1) and a condition of the form

$$
\int_{-1}^{1} \phi(t) d t=C .
$$

By use of (2.12) and the normal Gauss-Jacobi quadrature formula [6], the following system of equations is obtained:

$$
\begin{gathered}
\sum_{i=1}^{n} W_{i}^{n} \tilde{f}\left(t_{i}\right)\left[\frac{b}{t_{i}-x_{k}}+\pi k\left(x_{k}, t_{i}\right)\right]=g\left(x_{k}\right), \quad k=1,2, \cdots, n-1, \\
\sum_{i=1}^{n} W_{i}{ }^{n} \tilde{f}\left(t_{i}\right)=C / \pi \\
P_{n}^{(\alpha, \beta)}\left(t_{i}\right)=0, \quad i=1,2, \cdots, n, \\
P_{n-1}{ }^{(-\alpha,-\beta)}\left(x_{k}\right)=0, \quad k=1,2, \cdots, n-1 .
\end{gathered}
$$

(5.4) provides $n$ linear algebraic equations to determine $\tilde{f}\left(t_{i}\right), i=1,2, \cdots, n$.

5.2 The case of $\kappa=0$. For this case $\alpha=-\beta$. Here (2.12) provides $n$ equations:

$$
\begin{gathered}
\sum_{i=1}^{n} W_{i}{ }_{i}^{n} \tilde{f}\left(t_{i}\right)\left[\frac{b}{t_{i}-x_{k}}+\pi k\left(x_{k}, t_{i}\right)\right]=g\left(x_{k}\right), \quad k=1,2, \cdots, n, \\
P_{n}^{(\alpha, \beta)}\left(t_{i}\right)=0, \quad i=1,2, \cdots, n, \\
P_{n}^{(-\alpha,-\beta)}\left(x_{k}\right)=0, \quad k=1,2, \cdots, n .
\end{gathered}
$$

5.3 The case of $\kappa=-1$. For this case $\alpha>0$ and $\beta>0$. The solution must satisfy (5.1) and a consistency condition [3]

$$
\int_{-1}^{1} \frac{S(x)}{(1-x)^{\alpha}(1+x)^{\beta}} d x=0,
$$

where $S(x)$ is given by (2.8). By use of (2.1) the consistency condition is written as

$$
\sum_{i=0}^{p} B_{i} \int_{-1}^{1} P_{i+1}^{(-\alpha,-\beta)}(x)(1-x)^{-\alpha}(1+x)^{-\beta} d x=0,
$$

which is an identity. Use of (2.12) then yields $n+1$ equations:

$$
\begin{gathered}
\sum_{i=1}^{n} W_{i}^{n} \tilde{f}\left(t_{i}\right)\left[\frac{b}{t_{i}-x_{k}}+\pi k\left(x_{k}, t_{i}\right)\right]=g\left(x_{k}\right), \\
P_{n}^{(\alpha, \beta)}\left(t_{i}\right)=0, \quad i=1,2, \cdots, n, \\
P_{n+1}^{(-\alpha,-\beta)}\left(x_{k}\right)=0, \quad k=1,2, \cdots, n+1 .
\end{gathered}
$$

To solve (5.8) it is sufficient to choose only $n$ of the $n+1$ possible collocation points. This is consistent with the fact that in actual applications the extra equation is used to normalize the interval of integration.

6. Convergence of the method. For simplicity we consider the case of $\kappa=0$. The other cases may be treated in a similar way. First we construct an integral cquation satisfied by the interpolation polynomial $\tilde{f}(x)$. The determination of $\tilde{f}(x)$ is discussed in 
[7]. We define

$$
\begin{aligned}
\Phi(x) & =\tilde{f}(x) w(x), \\
\tilde{k}(x, t) & =\sum_{i=0}^{n-1} \sum_{i=0}^{n-1} k_{i, i} P_{i}{ }^{(-\alpha,-\beta)}(x) P_{i}{ }^{(\alpha, \beta)}(t), \\
\tilde{g}(x) & =\sum_{i=0}^{n-1} g_{i} P_{i}{ }^{(-\alpha,-\beta)}(x),
\end{aligned}
$$

where the coefficients $k_{i, i}$ and $g_{i}$ are determined by

$$
\begin{aligned}
\tilde{k}\left(x_{i}, t_{i}\right) & =k\left(x_{i}, t_{i}\right), \quad i, j=1, \cdots, n . \\
\tilde{g}\left(x_{i}\right) & =g\left(x_{i}\right), \quad i=1, \cdots, n .
\end{aligned}
$$

By using (2.12) and the standard Gauss-Jacobi quadrature formula [5], both of which are now exact, we obtain the integral equation

$$
a \tilde{\phi}(x)+\frac{b}{\pi} \int_{-1}^{1} \Phi(t) \frac{d t}{t-x}+\int_{-1}^{1} \tilde{k}(x, t) \tilde{\phi}(t) d t=\tilde{g}(x), \quad-1<x<1 .
$$

We form the difference between (5.1) and (6.6):

$$
a \epsilon(x)+\frac{b}{\pi} \int_{-1}^{1} \epsilon(t) \frac{d t}{t-x}+\int_{-1}^{1} k(x, t) \boldsymbol{\epsilon}(t) d t=\delta(x), \quad-1<x<1,
$$

where the error is defined by

$$
\epsilon(x)=\phi(x)-\phi(x)
$$

and the right-hand side is given by

$$
\delta(x)=g(x)-\tilde{g}(x)-\int_{-1}^{1}\{k(x, t)-\tilde{k}(x, t)\} \tilde{\phi}(t) d t .
$$

From (6.7) we see that if the integral equation (5.1) has a unique solution $f(x)$, then the approximate solution $\tilde{f}(x)$ converges uniformly to $f(x)$, if $\delta(x)$ converges uniformly to zero.

Actual error estimates may be obtained by regularizing (5.1) and (6.6) and then applying techniques described in [8]. Here the regularization will be shown. We consider the singular integral equation

$$
a \phi(x)+\frac{b}{\pi} \int_{-1}^{1} \phi(t) \frac{d t}{t-x}=h(x), \quad-1<x<1,
$$

and define the integral operator

$$
K_{x} h(x)=a \frac{h(x)}{w(x)}-\frac{b}{\pi} \int_{-1}^{1} \frac{h(t)}{w(t)} \frac{d t}{t-x}, \quad-1<x<1 .
$$

It is noted that if $h(x)$ is regular so is $K_{x} h(x)$. The solution $f(x)$ to $(6.10)$ may be evaluated by methods described in [2] and [3]. The result is

$$
\left(a^{2}+b^{2}\right) f(x)=K_{x} h(x), \quad-1<x<1 .
$$


By using

$$
h(x)=g(x)-\int_{-1}^{1} k(x, t) \phi(t) d t
$$

we obtain a Fredholm equation for the determination of $f(x)$ :

$$
\begin{aligned}
& \left(a^{2}+b^{2}\right) f(x)+\int_{-1}^{1} n(x, t) f(t) d t=K_{x} g(x), \\
& n(x, t)=w(t) K_{x} k(x, t), \quad-1<x<1 .
\end{aligned}
$$

The transformation of (6.6) yields a similar equation with degenerate kernel:

$$
\begin{aligned}
& \left(a^{2}+b^{2}\right) \tilde{f}(x)+\int_{-1}^{1} \tilde{n}(x, t) \tilde{f}(t) d t=K_{x} \tilde{g}(x), \\
& \tilde{n}(x, t)=w(t) K_{x} \tilde{k}(x, t), \quad-1<x<1 .
\end{aligned}
$$

The evaluation of $K_{x} \tilde{k}(x, t)$ and $K_{x} \tilde{q}(x)$ is easily performed by use of $(2.1)$, where $(\alpha, \beta)$ must be exchanged with $(-\alpha,-\beta)$. Error estimation for equations of the type (6.14) and (6.15) is described in detail in [8], chapter II.

7. Example. Consider the singular integral equation

$$
a p(x)-\frac{1}{\pi} \int_{-1}^{1} p(t) \frac{d t}{t-x}=0, \quad-1<x<1,
$$

which is solved under the condition

$$
\int_{-1}^{1} p(t) d t=P
$$

Eqs. (7.1) and (7.2) represent the plane elasticity problem consisting of a plane rigid stamp with sharp corners at $x= \pm 1$ sliding slowly in the negative $x$-direction on the surface of an elastic halfspace with a constant coefficient of friction $\eta . p(t)$ is the normal pressure, and $a$ is given by

$$
\begin{aligned}
a & =\frac{1}{2} \frac{1-2 \nu}{1-\nu} \eta \text { for plane strain, } \\
& =\frac{2-\nu}{4-\nu} \eta \text { for plane stress. }
\end{aligned}
$$

$\nu$ is Poisson's ratio [2].

In this problem the contact stress has integrable singularities at $x=-1 . \alpha$ and $\beta$ are then determined by

$$
\alpha=-1+\frac{1}{\pi} \arctan \frac{1}{a} \quad \beta=-\frac{1}{\pi} \arctan \frac{1}{a} .
$$

The solution is readily found to be

$$
p(x)=-\frac{P}{\pi} \sin (\pi \alpha)(1-x)^{\alpha}(1+x)^{\beta} .
$$


By means of the Fortran program described in [6] the constant factor in (7.5) was calculated for $\alpha=-0.34, \beta=-0.66$. For easy comparison $P$ was put equal to $-\pi /$ sin $(\pi \alpha)$. The results are given in Table I. The roots of the polynomials were determined with accuracy $10^{-10}$. From the results it is clear that singular terms of the form here treated can be calculated by use of the applied numerical routine.

\section{TABLE I}

Solution of (7.1) with $\alpha=-0.34, \beta=-0.66$ and $n=20$.

\begin{tabular}{ccc}
\hline \multicolumn{1}{c}{$t_{i}$} & $f\left(t_{i}\right)$ & $x_{k}$ \\
\hline 0.99570161 & 1.0000000 & 0.98941625 \\
0.96919398 & 1.0000000 & 0.95469739 \\
0.91882847 & 1.0000000 & 0.89647074 \\
0.84583484 & 1.0000000 & 0.81616593 \\
0.75200941 & 1.0000000 & 0.71575974 \\
0.63966222 & 1.0000000 & 0.59772435 \\
0.51155956 & 1.0000000 & 0.46496613 \\
0.37085573 & 1.0000000 & 0.32075402 \\
0.22101530 & 1.0000000 & 0.16863897 \\
0.06572782 & 1.0000000 & 0.01236655 \\
-0.09118299 & 1.0000000 & -0.14421527 \\
-0.24585350 & 1.0000000 & -0.29725094 \\
-0.39447517 & 1.0000000 & -0.44297222 \\
-0.53338846 & 1.0000000 & -0.57779094 \\
-0.65917283 & 1.0000000 & -0.69838740 \\
-0.76873102 & 1.0000000 & -0.80179206 \\
-0.85936525 & 1.0000000 & -0.88545862 \\
-0.92884347 & 1.0000000 & -0.94732641 \\
-0.97545332 & 1.0000000 & -0.98586879 \\
-0.99802548 & 1.0000000 & \\
\hline
\end{tabular}

\section{REFERENCES}

[1] F. Erdogan and G. D. Gupta, On the numerical solution of singular integral equations, Quart. Appl. Math. 30, 525 (1972)

[2] F. Erdogan, G. D. Gupta and T. S. Cook, Numerical solution of singular integral equations, in Methods of analysis and solutions of crack problems, G. C. Sih, ed., Noordhoff Leyden, 1973

[3] N. I. Muskhelishvili, Singular integral equations, Wolters-Noordhoff Publishing, Groningen, 1958

[4] F. G. Tricomi, On the finite Hilbert transformation, Quart. J. Math. Oxford 2, 199 (1951)

[5] M. Abramowitz and I. A. Stegun, Handbook of mathematical functions, Dover Publications, Inc., New York, 1965

[6] A. I. Stroud and Don Secrest, Gaussian quadrature formulas, Prentice-Hall, Inc., Englewood Cliffs, N. J., 1966

[7] S. Krenk, A note on the use of the interpolation polynomial for solutions of singular integral equations, to appear in Quart. Appl. Math.

[8] L. V. Kantorovich and V. I. Krylov, Approximate methods of higher analysis, Noordhoff, Groningen, 1964 